\title{
Le marqueur par exemple en français parlé au Cameroun
}

\author{
Simplice, Simeu \\ Lidilem, Université Grenoble Aples, France \\ simplice.simen@gmail.com
}

\begin{abstract}
Résumé
Le présent travail de recherche décrit et analyse le fonctionnement de par exemple dans un corpus de français parlé au Cameroun. Nous avons basé nos analyses principalement sur des enregistrements radiophoniques que nous avons complétés avec des écrits numériques du quotidien. Nous avons relevé deux des trois fonctions de par exemple qui sont présentes dans la littérature. Il s'est agi d'une part, du pointage qui opère au sein d'une catégorie (exemplification ou pointage intra-catégoriel) et, d'autre part, du pointage qui n'obéit à aucune catégorie mais qui s'intéresse à la subjectivité de l'énonciateur et à la manière dont elle transparait dans le discours, seul manque le pointage extra-catégoriel. De manière générale, l'utilisation du marqueur par exemple dans la variété de français parlé au Cameroun montre qu'il s'agit d'un continuum, c'est-à-dire que par exemple peut se déplacer du plan de l'énoncé vers celui de l'énonciation.
\end{abstract}

\begin{abstract}
The Marker par exemple in Cameroon Spoken French
This paper describes and analyses the functioning of par exemple in Cameroon spoken French. Based on radio programmes and some conversations gathered on Internet, findings show two out of the three functions of par exemple found in the literature. In the one hand, par exemple appears within a discourse category where it operates as a marker of exemplification whereas in the other hand par exemple does not obey to any discourse category, thus functions as a discourse marker. So, as a discourse marker, par exemple helps to express the subjectivity of the speaker and also indicates the way this is spread on his discourse. In a nutshell therefore, the use of par exemple in the variety of French spoken in Cameroon is a continuum; that is par exemple moves from the plan of "énoncé" to that of the enunciation.
\end{abstract}

\section{Introduction : Par exemple, sens et fonctions}

Le choix de par exemple comme objet de cette étude s'inscrit dans une perspective discursive où nous décrivons et analysons ses fonctions et ses usages dans la variété de français parlé au Cameroun. Quelles sont les rôles que jouent par exemple dans le dia-système du français? En existe-t-il un usage particulier à la communauté camerounaise?

Les études consacrées à par exemple en français que nous avons consultées (Vincent 1995, Vincent et al. 1993, Dostie 2002 et Rossari \& Jayez 2003) s'interrogent sur les différents sens de cette expression et sur le processus de pragmaticalisation ${ }^{l}$ qui caractérise cette particule. Partant des trois sens recensés généralement par les dictionnaires, les auteurs proposent différentes explications pour expliquer le passage du nom exemple à l'expression par exemple. Ce dernier peut prendre un sens assez éloigné de celui d'exemplification, donné en premier par le Petit Robert ( « considérons par exemple ce cas...»), le deuxième sens étant exclamatif ( ça par exemple !»), il marque l'étonnement ou la surprise et le troisième sens, courant au Québec, mais plus rarement attesté en Europe est dit « d'opposition ».

Dans le processus de pragmaticalisation qui fait passer un item nominal au statut de marqueur discursif (avec la perte au passage du déterminant et le gain de la préposition par), Dostie (2002:64) observe que le premier sens pragmatique reste très proche du sens de l'item lexical, mais, avec la poursuite du processus, le sens se démultiplie et on voit apparaitre des emplois qui s'éloignent de plus en plus du sens premier, tout en entretenant tout de même un lien avec lui. Il nous semble pouvoir résumer ce processus ainsi: le par exemple qui marque l'exemplification suppose que le locuteur crée une catégorie au sein de laquelle il va pointer un élément, tout en laissant entendre qu'il pourrait en pointer d'autres. De la valeur de pointage au sein d'une catégorie, illustrée par 
l'énoncé « Marie est pingre, par exemple elle ne s'achète jamais d'habits », où le locuteur instaure une catégorie de comportements susceptibles de recevoir l'étiquette " pingrerie», on peut passer à une simple mise en relief expressive, voulue par l'énonciateur (par exemple dans un commentaire évaluatif du genre : «par exemple, qu'est-ce qu'elle est pingre, Marie, elle ne s'achète jamais d'habits ! »), ou carrément à une valeur oppositive, où l'élément pointé ne fait pas partie de la catégorie instaurée au préalable: "Marie, elle est pingre, mais elle a acheté des habits par exemple !", énoncé qui peut être glosé par "malgré le fait que Marie soit pingre, elle a acheté des habits, ce n'est pas typique d'une personne qui n'aime pas dépenser son argent ». Nous pensons que nous pouvons rattacher ces trois types d'emploi à une seule fonction, en considérant, comme Rossari et Jayez (2003), qu'ils relèvent tous de la focalisation, cette dernière pouvant s'opérer à trois niveaux :

(1) le pointage qui opère au sein d'une catégorie comme dans l'exemplification

(2) le pointage qui opère en dehors d'une catégorie comme dans une situation indiquant un contraste ou une opposition

(3) la mise en exergue d'une attitude énonciative du locuteur comme la surprise, l'étonnement, le commentaire axiologique ou la simple mise en relief d'un topique

$\mathrm{Au}$ troisième niveau aucune catégorie n'étant instanciée, la focalisation est inscrite dans l'énonciation, cette dernière concerne le rapport qu'entretient le locuteur avec son énoncé. L'expression par exemple peut devenir un simple ponctuant du discours, parfois fréquemment réitéré (les puristes parleront de «tic de langage »), comme c'est le cas chez l'un des locuteurs de notre corpus.

Nous pouvons avancer qu'avec la pragmaticalisation, la fonction de pointage se déplace du plan de l'énoncé à celui de l'énonciation. Cela signifie que dans le cas du pointage intra- ou extra-catégoriel, on est sur le plan du référent à identifier (plan de l'énoncé), et dans le cas du pointage énonciatif, on est sur le plan de l'énonciation, c'est-à-dire du rapport du locuteur à son propre discours, lequel discours véhicule, comme nous l'avons déjà dit plus haut, différents degrés d'affectivité et d'expressivité. Il y a un glissement progressif d'un plan vers l'autre, ce qui explique le fait que certains emplois de par exemple aient été facilement classables et pas d'autres, puisqu'il s'agit d'un continuum.

Nous avons eu des exemples typiques, faciles à catégoriser et d'autres "à cheval" entre deux catégories, ce qui a créé une ambigüité au moment de l'analyse. Toutefois, nous avons considéré, comme Dubois (1969:109), que « l'ambigüité ne se situe pas du côté de l'émetteur, mais du côté du récepteur », et qu'il appartient à ce dernier de prendre une décision «qui est en réalité une recomposition » de l'énonciation, car il n'y a pas de symétrie entre l'émetteur et récepteur. Cela signifie que chaque analyse est une voie de désambiguïsation du contexte. En clair, « l'ambiguïté est la loi même d'un texte » ou d'un discours. Nous avons renoncé à ouvrir, comme l'a fait Vincent (1995:63), une catégorie regroupant des occurrences de par exemple «ambivalents » et nous avons pris la décision de classer nos occurrences suivant les différents emplois que nous avons indiqués plus haut, pour rappel, (1) l'exemplification, (2) le contraste ou l'opposition et (3) l'expressivité (ou la fonction énonciative). Nous aborderons désormais la présentation des données qui ont contribué à notre analyse ainsi que la présentation des résultats auxquels nous sommes parvenus.

\section{Présentation des données}

\subsection{Choix des données}

Les données de cette étude sont constituées avant tout d'enregistrements radiophoniques. Notre choix pour des discours radiophoniques comme données majeures a été motivé par plusieurs raisons :

- Les enregistrements radiophoniques sont d'une meilleure qualité

- Elles sont faciles à transcrire et l'interprétation nécessite uniquement l'ouïe (contrairement aux données multimodales).

- $\quad$ La radio est le média le plus divulgué en Afrique (Tudesq 2002).

- D'un point de vue pragmatique, ces discours illustrent à la fois la compétence de communication Chez Hymes et une compétence linguistique au sens chomskyen du terme.

Nous avons pris en compte trois genres radiophoniques dans la collecte des données de cette étude, mais seules les occurrences de deux genres, l'entretien («Spécial rencontre» - Corpus SR, "Parlons Femmes » Corpus PAF et «Politudes - Corpus POL) et le phone-in («De Midi à Quatorze heures » - DMQ), se sont avérées pertinentes pour nos analyses de par exemple. Nous avons recueilli les données dans trois radios : le poste national de la Cameroon radio and television (CRTV), Radio Tiemeni Siantou (RTS) et Tom broacasting corporation (TBC). Ces radios sont très appréciées par les habitants de Yaoundé la capitale du Cameroun où sont installées toutes les trois radios, dont les programmes couvrent presque l'ensemble des dix régions du Cameroun. 
Nous avons également tenu compte de la durée de l'implantation de la radio (qui était de cinq ans en 2010, année où nous avons collecté les données d'enquête), le taux d'audience et les langues utilisées (le français, l'anglais, les langues camerounaises, le pidgin-english et/ou le camfranglais). Nous avons eu accès à ces informations en interrogeant les animateurs des différentes radios que nous avons mentionnées et en consultant leurs grilles des programmes. Nous avons confirmé ces informations en écoutant nous-mêmes la radio et en menant un entretien semi-directif auprès des conducteurs de taxi (taximens) à Yaoundé, par exemple, à propos de leur(s) émission(s) préférée(s) dans chacune des radios concernées.

A ces données radiophoniques s'ajoutent, comme nous l'avons déjà annoncé plus haut, des écrits numériques du quotidien (ou encore discours électroniques médiés - DEM) collectés sur internet comme le journal en ligne (Corpus DEM - Journal TICMAG.net) et le réseau social Twitter (DEM-Twittter). Ces deux types de discours, qui abordent des thématiques variées comme le sport, la politique, les questions de la spiritualité et celles liées à pratique de la médecine traditionnelle, visent à réactualiser les données d'analyse et à toucher également des Camerounais qui vivent hors de leur pays. D'un point de vue purement méthodologique, ces types de données qui ne nécessitent plus à être transcrits ont le mérite de rendre compte d'autres caractéristiques que le chercheur doit prendre en compte lorsqu'il veut analyser l'oral. Venons-en à la distribution des occurrences de par exemple dans l'ensemble du corpus.

\subsection{La distribution de par exemple}

Comme le note Dostie (2002:151) : « on peut reprocher aux appellations « sens exemplaire », « sens oppositif », «sens expressif», etc. de tendre à "enfermer» en un seul mot notre compréhension des phénomènes fort complexes, plutôt que de l'élargir ». Par ailleurs, le découpage des exemples n'est pas toujours aisé non plus, il faut pouvoir tenir compte largement du contexte gauche et droit de l'occurrence pour parvenir à la classer de manière pertinente. Nous pensons tout de même que ce classement n'est pas vain, ne serait-ce que pour voir si les emplois « oppositifs » bien attestés au Québec se retrouvent au Cameroun. Il est évident que les occurrences de par exemple proviennent surtout des enregistrements radiophoniques et qu'elles soient peu nombreuses dans les DEM. Cela peut s'expliquer par la taille très réduite de ce sous-corpus comparée aux enregistrements radiophoniques (les DEM constituent 9000 mots, soit 10\% de l'ensemble du corpus qui compte 91000 mots).

Pour analyser les occurrences de par exemple, nous avons considéré (mais pas seulement) :

- les contextes gauche et droite (notamment le début ou la fin d'un énoncé ${ }^{2}$ ou d'une suite d'énoncés auquel est joint par exemple)

- les types de corpus (les discours sur internet ou les enregistrements radiophoniques)

- le nombre d'occurrences qui sont dues à un même locuteur

La distribution des 105 occurrences de notre corpus apparait dans le tableau ci-dessous. 54\% marquent l'exemplification, 46\% l'attitude énonciative et il n'y a pas d'occurrence avec valeur de contraste ou d'opposition :

Tableau : Place de par exemple selon la fonction (en \%)

\begin{tabular}{|c|c|c|c|}
\hline & Début & Final & Total \\
\hline exemplification & 32 & 25 & $54 \%$ \\
\hline Opposition & 0 & 0 & $0 \%$ \\
\hline $\begin{array}{l}\text { Expressivité (ou fonction } \\
\text { énonciative) }\end{array}$ & 24 & 24 & $46 \%$ \\
\hline Total & $53 \%$ & $47 \%$ & $100 \%$ \\
\hline
\end{tabular}

Source : données de l'étude

Malgré l'absence de par exemple à valeur oppositive, il nous semble prématuré de tirer une conclusion sur l'existence ou non de cette fonction d'opposition en français parlé au Cameroun, à partir des seules données de notre étude. Des études plus importantes et plus diversifiées permettront de confirmer ou d'infirmer ces résultats qui montrent la non existence de cette valeur oppositive dans la variété de français parlé au Cameroun. Cela signifie que cette fonction d'opposition reste, tout au moins pour l'instant, caractéristique du seul français québécois, comme l'indiquent d'ailleurs les linguistes québécoises que nous avons mentionnées plus haut ( $c f$. Vincent et al. 1993, Vincent 1995 et Dostie 2002).

Dans notre corpus par exemple à tendance à occuper le début de l'énoncé et dans cette position c'est la valeur exemplaire qui est la catégorie la plus mise en avant (32/56, soit 57\%) avant l'expressivité (25/56, soit 43\%). En 
fin d'énoncé les occurrences de par exemple sont également réparties de façon équilibrée soit 51\% (25/49) pour la fonction exemplaire et $49 \%$ (24/49) pour la fonction expressive. Nous n'avons relevé que six occurrences de par exemple dans notre sous-corpus DEM (pour rappel discours électronique médié), dont deux occurrences qui sont tirées d'échanges informels (sur Facebook et Twitter) et quatre occurrences tirées d'un entretien publié dans la presse en ligne (TICMag.net). Ces occurrences relèvent toutes de la fonction exemplaire, et elles sont réparties de manière équilibrée entre le début et la fin des énoncés dans lesquels elles apparaissent. Les occurrences relevées dans les émissions d'entretien radiophonique représentent 70\% (73/105) des occurrences de l'ensemble de notre corpus contre $25 \%(26 / 105)$ pour les talk-shows et les phone-in et 5\% (6/105) pour les DEM.

Les trois émissions d'entretien radiophonique ('Parlons femmes' - PAF, Politudes - POL, 'Spécial rencontre' - SR) rassemblent la majorité des occurrences de par exemple dans notre corpus. L'émission 'Quatre S' (QS1) fait exception puisqu'elle ne contient aucune occurrence alors qu'il s'agit également d'un entretien. L'émission SR1 comprend cinq occurrences de par exemple dont quatre produites par LK, l'invité, et une seule émise par l'animateur FB. Dans POL1, on relève cinq occurrences de par exemple dont quatre sont produites par le locuteur interviewé, DSN, et une par la journaliste CB. L'émission POL2 contient neuf occurrences de par exemple, toutes produites par la personne interviewée, GAA. Enfin dans l'émission PAF la personne interviewée ( $\mathrm{SAB}$, un universitaire) utilise quarante-neuf fois par exemple (sur les cinquante-deux occurrences de la transcription). A partir de ces observations, nous faisons l'hypothèse que l'emploi de par exemple dans une émission d'entretien radiophonique est plus souvent du ressort de la personne invitée que du ou de la journaliste (et/ou l'animateur/l'animatrice). Venons-en à l'analyse des différentes fonctions.

\section{Par exemple d'exemplification (ou la preuve par l'exemple)}

Le par exemple d'exemplification permet, nous l'avons dit plus haut, d'opérer un pointage intra-catégoriel. Rossari et Jayez (2003) parlent de processus discursif de particularisation/généralisation dans lequel par exemple joue pragmatiquement le rôle de la preuve qui valide une assertion. Rossari et Jayez (2003:463 sq.) montrent que cette validation de l'assertion au moyen de par exemple peut s'établir d'au moins quatre manières différentes : par un mouvement qui va de l'abstrait au concret (1a), d'une pluralité à une singularité (1b), d'une possibilité à une réalisation effective (1c) et d'une indication non spécifique à une illustration (1d) :

(1) [exemples construits donnés par Rossarie et Jayez (2003:463 sq.)]

(a) «Il aime les belles voitures, par exemple (il aime) les Audis. »

(b) «Il a émis des objections contre plusieurs théories, par exemple contre la théorie du big bang. »

(c) «Il y a des difficultés, par exemple sociales, qu'on ne peut pas régler avec une simple loi. »

(d) «Il a eu tous les ennuis, par exemple il s'est cassé le bras ».

Ces quatre procédures de focalisation se retrouvent dans notre corpus avec une fréquence plus ou moins grande. Dans l'émission SR1 où FB reçoit en entretien LK presqu'octogénaire, les occurrences de par exemple relèvent d'au moins trois des quatre procédures exemplifiées ci-dessus : le mouvement qui va d'une pluralité à une singularité, d'une indication non spécifique à une illustration et d'une possibilité à une réalisation effective.

\subsection{La preuve par l'exemple: de la pluralité à la singularité}

Dans l'exemple ci-dessous (extrait 2) LK revendique son appartenance à la communauté linguistique des «basaa ${ }^{3} »$ du Cameroun, ce qui avalise sa capacité à pouvoir traiter la maladie provoquée par le $n s o n g^{4}$ (un mauvais sort et qui se caractérise par le dessèchement du corps humain) :

(2) [Corpus SR1, 89-90]

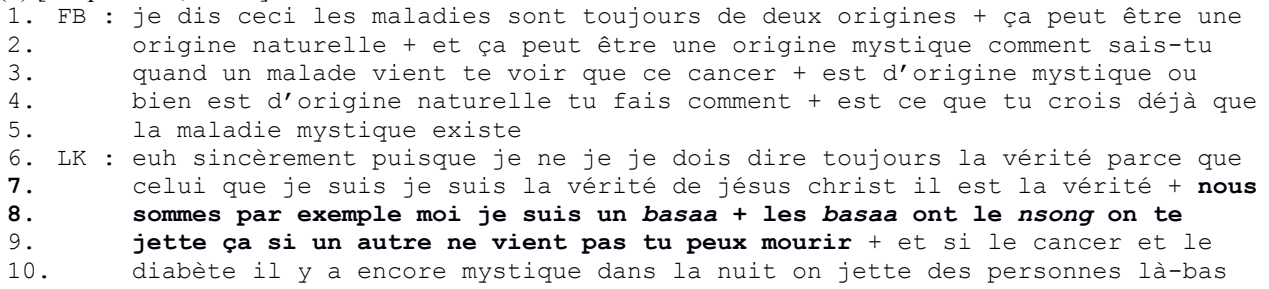

Dans l'échange ci-dessus, la valeur exemplaire obéit au mouvement de la pluralité ("nous sommes") à la singularité ("par exemple moi je suis"). Le fait de débuter l'énoncé par "nous sommes" valide en premier lieu la 
catégorie communautaire les Basaa: le nsong est un sort qui est jeté par un Basaa et ne peut être traité que par un Basaa. Le mouvement de la preuve va de la pluralité à la singularité, le pointage du référent va de " nous » à " moi je », et s'effectue de manière anaphorique dans la mesure où « nous » renvoie au syntagme nominal "les basaa ». L'énoncé « par exemple moi je suis un basaa» est une incise qui vient expliciter le nous initial reformulé ensuite par les basaa comme le montre la mise en grille ${ }^{5}$ ci-dessous :

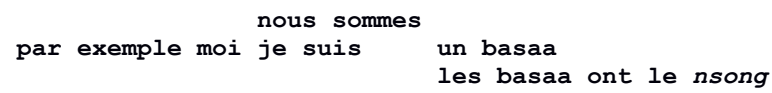

L'exemple ci-dessous illustre également ce mouvement de la pluralité à la singularité. Il s'agit d'un extrait de l'entretien donné par un jeune webmaster ${ }^{6}$ Camerounais au journal TIC MAG (TM dans l'échange ci-dessous) publié sur internet. AO, étudiant à Shanghai, explique la motivation qui est à la base de la création d'un nouveau réseau social, $\mathrm{Mycombi}^{7}$.net, destiné exclusivement aux Camerounais qui se trouvent partout dans le monde, dont il est co-fondateur (avec un autre étudiant Camerounais expatrié lui aussi à Shanghai) :

(3) [Corpus DEM, journal en ligne (TIC MAG.net)]

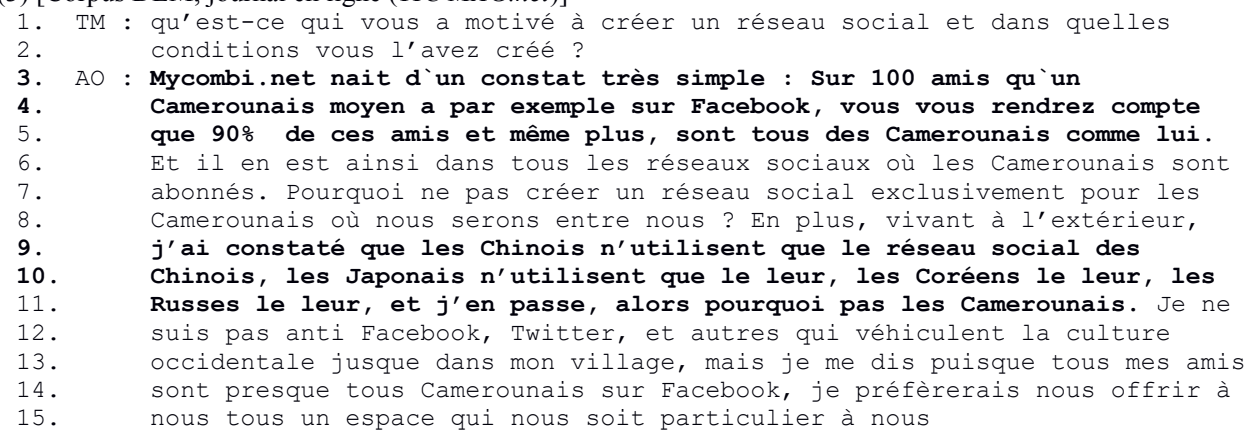

L'introduction de par exemple permet d'instancier à posteriori la catégorie des réseaux sociaux (pluralité) et de pointer sur Facebook (singularité). La catégorie va être verbalisée un peu plus loin dans le discours à travers l'énumération des différents réseaux sociaux. Bien sûr, le discours laisse entendre qu'on part d'une pluralité de réseaux sociaux, dans laquelle on pointe un peu au hasard Facebook. En fait, il s'agit d'une stratégie argumentative car $\mathrm{AO}$ sait que ce réseau social est le plus utilisé à travers le monde et il souhaite que Mycombi.net le concurrence bientôt en devenant le réseau des Camerounais, y compris au sein de la diaspora.

L'exemple suivant est un échange entre des internautes, certainement africains, où les scripteurs (STS et KC) partent à nouveau d'une pluralité (les équipes de football africaines) pour resserrer sur l'équipe du Cameroun :

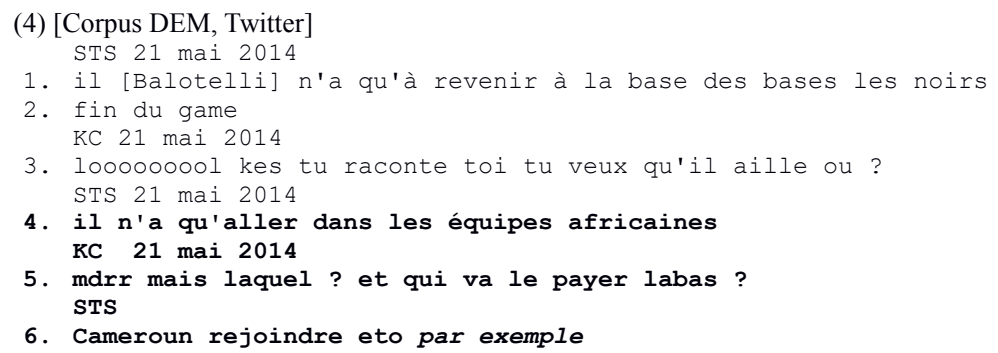

L'occurrence de par exemple dans l'extrait ci-dessus sert aussi de marqueur de clôture du discours, en plus de sa fonction d'exemplification qui nous intéresse ici. Cet exemple 4 montre, comme nous l'avons déjà indiqué plus haut, que certaines occurrences de par exemple de notre corpus peuvent appartenir à plus d'une catégorie. La section suivante présente le mouvement d'exemplification qui va d'une indication non spécifique à une illustration. 


\subsection{La preuve par l'exemple: de l'indication non spécifique à l'illustration}

Dans l'extrait 5 ci-dessous, par exemple fait le lien entre la catégorie assez large de "spécialisation" introduite par FB (ligne 3) et une nouvelle sorte de cancer chez la femme (ligne 9) :

(5) [Corpus SR1, 139-140]

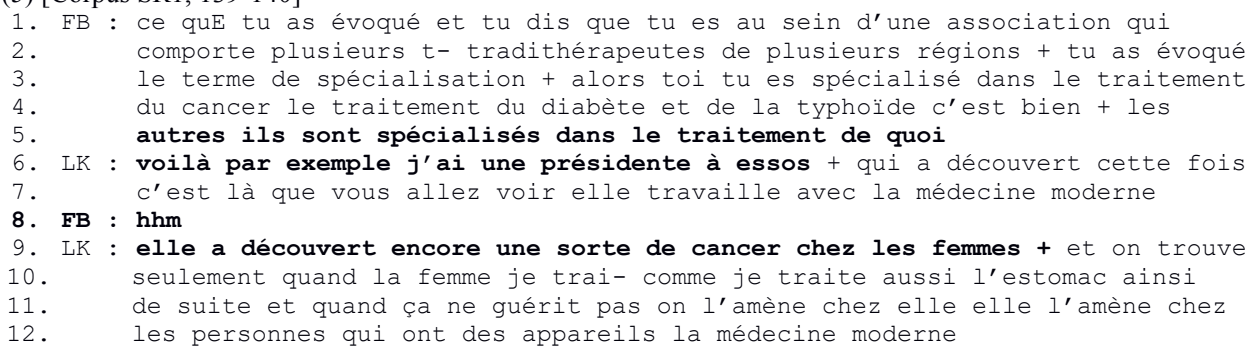

Il est intéressant de constater que LK ne donne pas un exemple de pratique traditionnelle typique, puisque son exemple concerne une collègue qui travaille « avec la médecine moderne ». Dans ce cas, l'illustration n'est justement pas un exemple de pratique traditionnelle, mais le par exemple du début de l'intervention permet à LK d'enchainer thématiquement sur l'objet qui est posé dans la question de FB.

Les extraits que nous avons analysés dans les deux sections ci-dessus proviennent d'intervention réactive dans laquelle la personne interviewée répond à une question de l'animateur. Comme nous l'avons dit plus haut, ce sont la grande majorité des occurrences du corpus d'entretien (aussi bien radiophonique que ceux issus d'internet). Par exemple est aussi utilisé dans une séquence initiative (autrement dit par l'interviewer). L'extrait 6 illustre un cas d'exemplification qui consiste à passer d'une possibilité à une réalisation effective.

\subsection{La preuve par l'exemple: d'une possibilité à une réalisation effective}

Dans l'exemple ci-dessous (extrait 6) par exemple apparait dans une intervention de l'interviewer FB qui cherche à persuader l'interviewé d'adopter une autre façon de faire avec les paiements de ses patients :

(6) [Corpus SR1, 156-161]

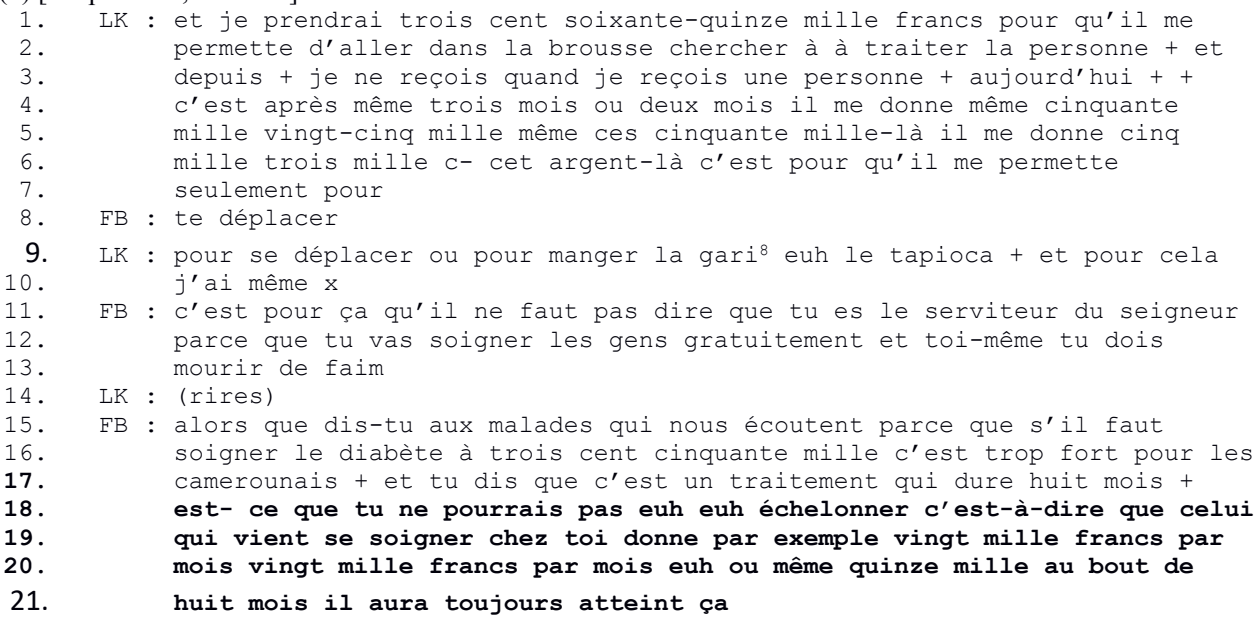

La séquence de persuasion initiée par FB dans l'extrait ci-dessus débute à la ligne 11 et s'achève à la ligne 21. FB utilise par exemple à la ligne 19 pour exposer plusieurs possibilités liées au règlement des frais de traitement des maladies ; cette énumération suit l'énoncé : « est-ce que tu ne pourrais pas euh euh échelonner». 
Les occurrences de par exemple que nous avons analysées jusqu'à présent montrent un pointage sur le plan de l'énoncé, le dit, à travers l'identification du référent. La description qui va suivre s'intéresse à la série constituant des occurrences de par exemple qui fonctionnent dans le dire, montrant ainsi le glissement de par exemple du plan de l'énoncé au plan de l'énonciation.

\section{Par exemple d'expressivité}

Les occurrences de par exemple qui marquent l'expressivité sont celles qui font ressortir la subjectivité du locuteur. Lors du processus d'énonciation, par exemple joue le rôle de ponctuant (c'est-à-dire de marqueur discursif, $c f$., entres autres, Vincent 1982 et Dostie 2007). Les deux premières séquences que nous traitons (7 et 8) relèvent toutes les deux du genre de l'explication et les occurrences de par exemple qui y apparaissent appartiennent davantage au plan de l'énonciation qu'à celui de l'énoncé.

L'extrait 7 ci-dessous est tiré de l'émission radiophonique DMQ où les auditeurs appellent pour apporter une opinion en vue de solutionner le problème d'un autre auditeur, problème qui a été relayé à l'antenne par l'animateur sous forme de thématique du jour. L'auditrice au téléphone GE revient à l'antenne après un précédent appel pour donner à nouveau des conseils à un précédent auditeur (de ne plus tromper sa femme, en l'occurrence) :

(7) [Corpus DMQ1, $399-421]$

1. GB oui germaine avec une des idées nouvelles apparemment

2. GE : oui je voudrais simplement dire à ce monsieur que le plus souvent le

3. bonheur d'un couple quI de temps en temps se déchire n'est pas souvent très

4. loin ça peut être son son ami le plus proche

5. GB : hhm

6. GE : ça peut être son frère le plus proche

7. GB : oui

8. GE : oui donc euh euh le bonheur de ce couple qui se déchire n'est pas souvent

9 . très loin il suffit d'un d'une moindre volonté

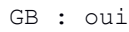

Le par exemple de la ligne 21 appartient au plan de l'énonciation dans la mesure où il ne cherche pas à pointer une des possibilités qui s'offrent à un couple en crise mais rend explicite (encore que sous une forme périphrastique) une information qui est présente implicitement depuis le début de l'échange (à savoir que l'appelant a une relation extraconjugale). Cette occurrence de par exemple qui se trouve en fin d'énoncé permet un premier mouvement de clôture interactif de l'échange, initié par le régulateur « hhm » de GB (ligne 20), et immédiatement suivie de deux «tout à fait» (ligne 22). Ce mouvement de clôture est bien saisi par GE qui propose une récapitulation de son propos, en produisant elle aussi deux marqueurs de clôture, "c'est tout " (ligne 24) et « voilà » (ligne 25). Cette conclusion est acceptée par GB qui émet à son tour un « merci » à la ligne 29.

L'exemple suivant (extrait 8) est issu de l'émission radiophonique PAF. Dans cette émission SAB et CPT évoquent la place de la femme dans la société camerounaise et SAB est chargé en tant qu'expert de proposer des solutions pour améliorer les conditions de vie des femmes camerounaises, y compris au travail et lors des manifestations relatives à leur statut de femme. L'extrait débute avec une contre-proposition de la journaliste qui 
enchaine sur une intervention de SAB laissant entendre que le mouvement des femmes entre le village et la ville va toujours du premier à la seconde :

(8) [Corpus PAF, 107-108]

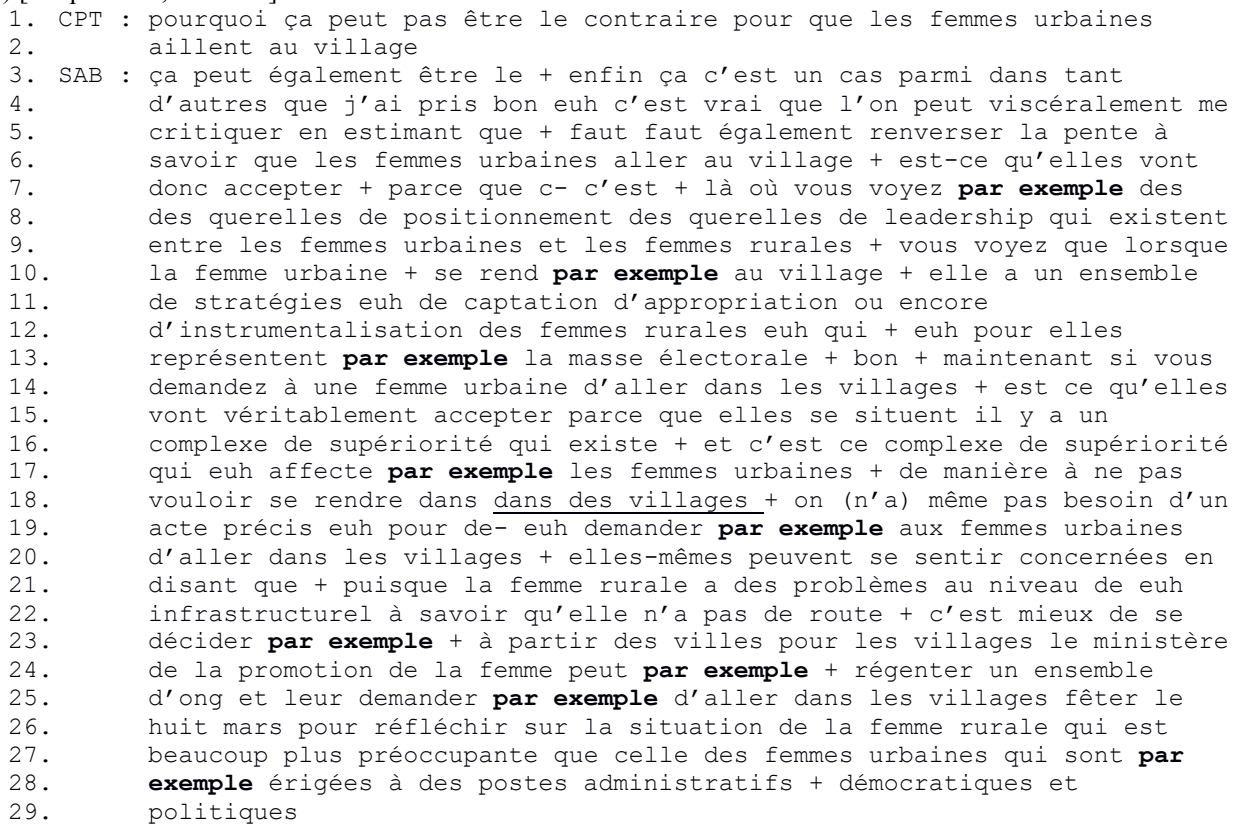

Par exemple est utilisé ( 9 fois) dans la longue explication fournie par SAB suite à la relance produite par CPT sur le thème du mouvement des femmes entre la ville et le village. Les occurrences régulières de par exemple illustrent bien sa fonction de ponctuant du discours. Ils constituent des indices explicites d'une difficulté à discourir sur le délicat problème des différences ville-campagne, plus précisément sur les rapports de domination entre les femmes urbaines et les femmes rurales. La multiplication des occurrences de par exemple dans cet exemple (extrait 8) joue un rôle de facilitateur pour $\mathrm{SAB}$, il gagne du temps afin de trouver le mot adéquat dont il a besoin pour expliquer son raisonnement en ménageant la face des éventuelles auditrices de la campagne. Cela veut dire que l'emploi de par exemple n'est pas simplement un tic de langage chez cet universitaire mais qu'il l'aide à construire les différences catégorielles entre femmes urbaines et femmes rurales.

Nous allons prendre un dernier extrait permettant d'illustrer la fonction de par exemple au plan de l'énonciation, cette fois en décrivant et analysant une séquence qui relève de l'évaluation ou du commentaire. L'extrait 9 est issu de l'émission POL. Le gouverneur GAA qui est reçu en entretien par BTN s'indigne du comportement de son ministre de tutelle: ce dernier aurait abusé de son pouvoir pour autoriser la mutation d'un agent à l'intérieur d'une région administrative alors que cette compétence de mutation intra-régionale relève de la seule compétence du gouverneur (le ministre ayant le droit de faire uniquement des mutations interrégionales) :

(9) [Corpus POL2, 83-84]

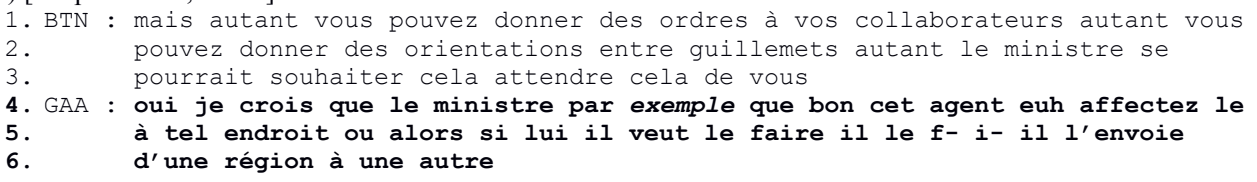

Par exemple apparait ici au sein d'un marqueur de reformulation paraphrastique complexe «par exemple que bon » qui entraine un changement de topique. GAA préfère parler de l'agent plutôt que du ministre, ce qui révèle d'une stratégie de prudence. 


\section{Bilan conclusif}

Au terme de notre analyse des occurrences de par exemple, nous faisons au moins deux réflexions principales. La première montre que le fonctionnement de par exemple dans notre corpus permet de dire qu'il n'y a pas de spécificité camerounaise en la matière. Certains usages que nous avons analysés ci-dessus montrent que par exemple joue également le rôle de marqueur discursif qui permet au locuteur de discourir sur des thématiques délicates et qu'il procède par des stratégies de rattrapage, d'évitement comme la périphrase, bref par l'expressivité tout en faisant preuve de prudence.

La deuxième réflexion est en rapport avec la fonction contrastive que peut revêtir les par exemple d'expressivité, sans que ce contraste aille jusqu'à une valeur d'opposition locale, comme c'est le cas dans le parler québécois.

\section{Références bibliographiques}

Badiou-Monferran, C et Buchi, E (2012). «Plaidoyer pour une désolidarisation des notions de pragmaticalisation et de grammaticalisation », dans Neveu, F. et al. (éds), Actes du $3^{e}$ Congrès Mondial de Linguistique Française, France : Institut de Linguistique Française, 127-144.

Dostie, G. (2002). « L'exemplarité de par exemple. Un cas de pragmaticalisation en français québécois». French Language Studies, 12, 149-167.

Dubois, J. (1969). «Énoncé et énonciation ». Langage, 13, 100-110.

Ethnologue (2015). Languages of the World, 18th edition : https://www.ethnologue.com/ethnoblog/m-paullewis/welcome-18th-edition.

Rossari, C. et Jayez, J. (2003). « Par exemple: une procédure d'exemplification par la preuve », dans Combettes, B., Schnedecker, C. \& Thiessen, A. (éds), Actes du colloque de Metz, Ordre et distinction dans la langue et le discours, Paris: Honoré Champion, 461-478.

Simeu, S. (2016). Le français parlé au Cameroun : une analyse de quatre marqueurs discursifs, là, par exemple, ékyé et wéè. Thèse de doctorat. Université Grenoble Alpes.

Tudesq A.-J. (2002). L'Afrique parle, l'Afrique écoute. Les radios en Afrique subsaharienne. Paris: Karthala.

Vincent, D. 1995. « Remarques sur par exemple en français Québécois ». Le français modern, 63 (1), 57-73.

Vincent, D., Votre, Sebastião et Laforest, Marty (1993). " Grammaticalisation et post-grammaticalisation ». Langues et linguistique, 19, 73-100.

\section{Protocole de transcription}

L1, L2, L3, Ln, pour noter les locuteurs (nous avons remplacé ces symboles par les initiales

$\begin{array}{ll}\text { oui } & \text { les chevauchements } \\ + & \text { pause courte }(0-1 \text { seconde) } \\ ++ & \text { pause moyenne }(1-2 \text { secondes }) \\ --- & \text { pause longue }(2-3 \text { secondes })\end{array}$

explication $\}$ interruption assez longue du discours (enregistrement non coupé)

(0.) pause en fin du tour de parole (le nombre de seconde est indiqué)

(rire), (toux)

rapidité de l'enchainement entre deux tours de parole

$\$ \$ \$$ \{explication coupure de l'enregistrement

$\mathrm{x} \quad$ syllabe inaudible

xxx suite de syllabes inaudibles

- $\quad$ troncation

/, / multi-transcription, alternance auditive

/,Ø/ quelque chose ou zéro : et /alors, Ø/ dans ce que on s'en débarrasse

( ) transcription incertaine (hésitation orthographique) : on (n') en parle

CAPITALS : lettre, syllabe ou mot accentué ou un volume fort de la voix

- $\quad$ Les appels de notes pour souligner, en bas de pages, les faits prononciation et de bruits

1 Badiou-Monferran et Buchi (2012:127-128) plaident pour une désolidarisation des notions de pragmaticalisation et de grammaticalisation. Ces deux auteures récursent l'idée selon laquelle la pragmaticalisation constitue un cas particulier de la grammaticalisation et soutiennent que l'une n'est pas incluse dans l'autre mais qu'il s'agit de deux phénomènes différents. 
${ }^{2}$ Une séquence orale ou une suite finie d'unités de la chaine parlée, délimitée par deux périodes de silence

${ }^{3}$ On dit aussi basa-bakongo, ce qui permet de différencier cette communauté présente au Cameroun des basaa vah, communauté linguistique présente en Sierra Leone mais surtout au Libéria (Ethnologue 2015). La langue basaa du Cameroun (Afrique centrale) et celle parlée en Afrique de l'Ouest font partie de la famille NigerCongo, famille de langues la plus représentée en Afrique subsaharienne.

${ }^{4}$ Le $n s o n g$ est une pratique mystique au Cameroun.

${ }^{5}$ Analyse syntaxique basée sur le modèle aixois représenté par Claire Blanche-Benveniste dont le but est de transcrire le français parlé en tenant compte des configurations séquentielles comme les brides, les tenues en ménoires (qui se manifestent par les phénomènes d'anticipation ou les digressions), les symétries et les rythmes.

${ }^{6}$ Cet anglicisme est traduit par webmestre au Québec et en France par administrateur de site ou administrateur de serveur mais nous préférons cet anglicisme parce qu'il est plus courant chez les locuteurs Camerounais.

7 Nom (masc. sing.) issu de la composition entre l'adjectif possessif anglais my et combi qui signifie « ami/amie » en pidgin-english camerounais, et que nous suspectons qu'il serait issu de l'anglais chum ou plus probablement de chummy qui signifient respectivement « ami » et « amical » en français.

${ }^{8}$ Tapioca en pidgin-english camerounais 